\title{
The Role of Footwear in Rehabilitation: A Review
}

\author{
H Branthwaite, N Chockalingam
}

\section{Citation}

H Branthwaite, N Chockalingam. The Role of Footwear in Rehabilitation: A Review. The Internet Journal of Rehabilitation. 2009 Volume 1 Number 1.

\begin{abstract}
Whilst footwear is primarily used to protect the foot from cutaneous injury, its use as a therapy in rehabilitation is becoming more significant as shoes can be selected and modified to meet the clinical needs of a patient.This paper provides a general overview to the uses of footwear in the rehabilitation of patients with diabetic foot complications, rheumatoid arthritis, osteoarthritis and the elderly. Furthermore, it highlights the role footwear plays in improving function and performance. Therapeutic footwear and footwear adaptations are frequently used within rehabilitation to accommodate joint deformities, modify plantar pressure distribution, alter gait patterns and improve stability and balance. However there are limited footwear choices for common foot health problems leading to persistent problems associated with fit and comfort. The evolution of a bespoke shoe that is fit on the high street would provide an effective therapy for the masses.
\end{abstract}

\section{INTRODUCTION}

Previous reports have indicated that rehabilitating a patient to restore function can be time limiting (Nolan et al, 2000). The role of footwear in providing support to improve performance by addressing restraints associated with mobility is known amongst rehabilitation professionals. Whilst the primary function of footwear is to protect the foot from cutaneous injury, it plays a crucial role as a therapeutic device. It is often used as (i) a part of the rehabilitation process of patients in the recovery of musculoskeletal injury, (ii) a pressure relieving device in complex diabetic and rheumatoid foot complaints and (iii) an aid to accommodate congenital and developmental deformity (Janisse and Janisse, 2008).

Therapeutic footwear can be prescribed with modifications and adaptations added to a standard shoe or the shoe can be manufactured bespoke to the needs of the patient. A standard high street shoe is comprised of several variable components that will influence the style, fit and comfort (Figure 1). Fashionable shoes that are worn as an accessory with little function, consist of different shapes for each section that can considerably alter the style and fit of the footwear. Similarly, in high performance sports shoes the components of the shoe are modified depending on the sport and material properties of the sole can enhance performance by improving shock absorbency. However in rehabilitation and therapeutic footwear, the components of a standard shoe are modified to meet the needs of the patient. A frequent requirement being increased depth and width of the toe box (Williams and Nester, 2006).

\section{Figure 1}

Figure 1: Components of a shoe

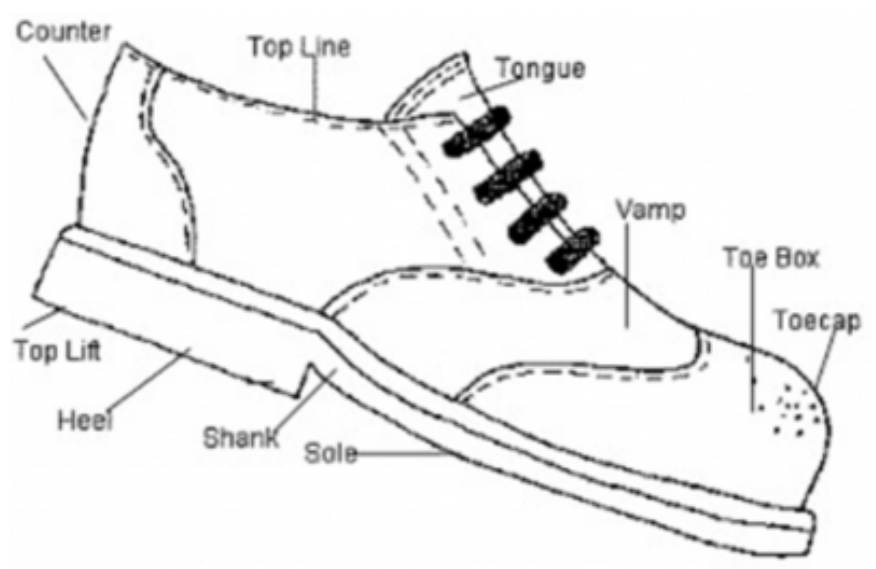

The perception of orthopaedic and prescription footwear can sometimes be deemed as unacceptable to patients as the current styles and shoe modifications are undesirable and high street shoes are often utilised in favour (Williams and Nester, 2006).

\section{ORTHOPAEDIC FOOTWEAR}

A Rocker sole is the most commonly prescribed orthopaedic modification for the benefit of reducing plantar pressure and removing the propulsive phase of gait (Hutchins et al, 2009, Fuller et al, 2001). Whilst reduction in plantar pressure is a desirable outcome for management of the rheumatoid foot, a 
previous study (Hennessey et al, 2007) evaluated the reduction in plantar pressure between an orthopaedic shoe and a normal running shoe in patients who had forefoot pain associated with rheumatoid arthritis. The results indicated that the running shoe was more effective in reducing plantar pressure and accepted as comfortable by the participants. These running shoes are normally made to standard widths and depths and hence the toe deformities often seen in rheumatoid patients may not be accommodated within offthe-shelf running shoes. However, running shoes are still a popular choice for management of foot pain in the initial phases of the disease when gross joint deformities have not developed (Hennessey et al, 2007). While, ankle fusion and severe ankle osteoarthritis causing limited ankle motion respond well with the use of rocker sole modifications, previous reports indicate that altered heel strike kinetics and an increase in toe off plantarflexion shortens the stride length and thus reduces the required motion at the ankle joint (Myers et al, 2006).

Although the use of rocker shoe modifications demonstrate desired clinical effects, previous investigations (Wu et al, 2004 and Long et al, 2007) have highlighted an increase in knee and hip flexion. Furthermore, the joints are in a flexed position for a longer period throughout the gait cycle whilst wearing a shoe with a rocker sole modification. While, potential problems that could arise from this change has not been recorded in the population who wear prescription footwear, recent research indicates increased joint flexion and time spent in a flexed position as influential factors in the development of musculoskeletal overuse injuries (Willems et al, 2006).

More complex acquired and congenital deformities, as seen in conditions such as chronic osteoarthritis of the toes, foot collapse or club foot, can require significant shoe modifications to support the foot and rebalance any asymmetry (Tyrell and Carter, 2009). Furthermore, accommodations for limb length discrepancy and fixed joint positioning can be implemented on the sole of the shoe or with in the structure of the shoe. These adaptations and modifications that are made are frequently are bespoke in design and meet the clinical needs of the patient to improve their stability and function whilst walking (Tyrell and Carter, 2009).

\section{DIABETIC FOOTWEAR}

The key aim for the use of footwear in the rehabilitation of diabetic patients is to provide shoes that improve the healing and prevention of plantar and dorsal foot ulcerations. Pressure relief has been highlighted as an important component in healing of diabetic ulcerations (Piaggessi et al, 2003). The use of appropriate footwear to reduce dorsal and plantar pressure the healing time for foot ulceration improves reducing the risk of further complications. Furthermore, forefoot off loading shoes (Figure 2) are normally utilised in initial management of ulcer treatment as they accommodate bulky dressings and reduce plantar pressure. However, they are not perceived as comfortable shoes which could influence compliance of treatment (Bus et al, 2009).

Once healing of any ulceration is achieved, the use of therapeutic/orthopaedic footwear to prevent further ulceration and tissue loss is used in the rehabilitation of patients (Bus et al, 2008 (a)). These modifications may include the use of a rocker sole, the positioning of which could be reflective of the position of any ulceration and the desired effect that is required (Hutchins et al, 2009).

Bespoke changes to the toe box, heel counter and top line are often used to improve fit and comfort of the shoe.

\section{Figure 2}

Figure 2: A Forefoot off Loading Shoe

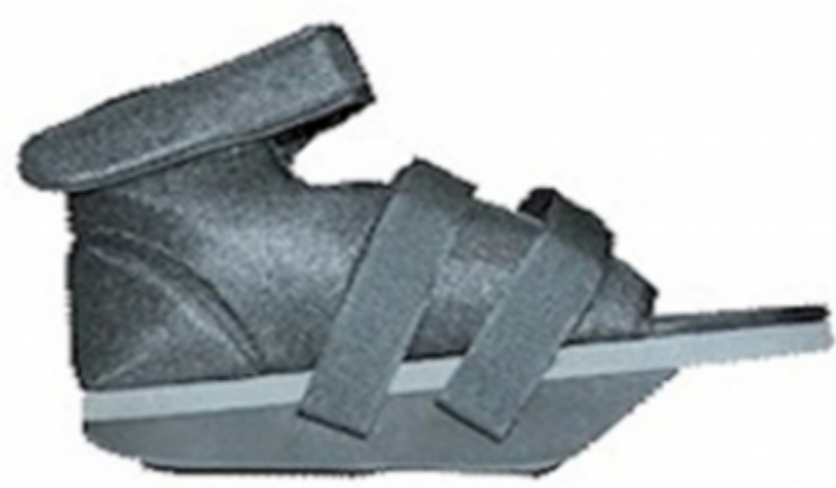

The use of footwear in diabetic foot ulceration is widely adopted with in clinics. However a recent review (Bus et al, 2008 (b)) concluded that although the effects of footwear looked promising, there was a clear paucity of sound studies to support the use of footwear in the rehabilitation of diabetic foot ulceration.

\section{PHYSIOLOGICAL EFFECTS OF PRESCRIPTION FOOTWEAR}

Previous investigations have documented the use of footwear to change gait kinematics and kinetics as well as muscle function and oxygen consumption (Luo et al, 2009). While, these principles are traditionally used to alter gait in a 
pathological population they have now become a household feature with consumers being able to influence and alter normal function with therapeutic footwear in the form of commercial branded shoes (MBT, Sketchers Shape-ups, Chung shi, FitFLop and Xsensible stretch). The explosion of products to improve posture and alter gait have been a success in the health and fitness market, with sales of the MBT shoe and Fit Flop soaring since their launch and marketing. However there is little scientific evidence to support the health benefits of wearing therapeutic footwear in the long term without an existing pathology.

Romkes et al, (2006) found changes in tibialis anterior and gastrocnemius muscle activity when wearing the MBT shoe and concluded that the footwear be utilised as a part of a strengthening exercise regime for these muscles. Similarly, Nigg et al, (2006) observed beneficial changes in tibialis anterior activity that supported the therapeutic use of rocker soled shoes.

The extent to the benefits of persistent use of such shoes is not understood or documented and could potentially be detrimental with alteration in muscle activity and gait patterns. There is a possibility that these changes become irreversible and normal high street footwear unusable.

\section{FOOTWEAR AND FALLS}

Ambulation in an effective and safe manner plays an important role for the advanced rehabilitation of patients whose gait has been affected by their health and in the elderly population. Balance and stability are often key parameters that can influence how rapid the progress of normal walking is achieved. Stability of footwear can influence the balance of the patient. Menant et al, (2008) highlighted that the composition of the sole of a shoe caused changes in the base of support and recommended that elderly people who were at risk of falling should wear shoes that had a low collar and standard sole stiffness, with or without treads to increase stability. However Decker et al, (2009) found that there was a reduction in coordination with lower traction (grip) of the shoe. This would then increase the risk of falls. It is unclear which is more beneficial for preventing falls as an increase in traction may induce a stumble in an elderly patient whose gait is compromised towards a shuffle and a reduced traction would aid this shuffling gait.

Nurse et al, (2005) recommended the use of a textured insole in therapeutic footwear as they observed an improvement in sensory feedback and reduced muscle activity, thus improving proprioception and stability. It would be a suitable conclusion to implement a combination; incorporating a textured insole into footwear and having a sole with moderate traction to improve the stability and function.

\section{FOOT HEALTH}

Foot health problems are prevalent in the elderly population with females suffering more than men (Benvenuti et al, 1995). Advanced arthritic toe deformities including hallux valgus, hammer toe, and joint subluxation cause problems with standard toe box depths and widths. High street footwear that accommodates these problems are normally difficult to obtain causing an increased tendancy for ill fitting shoes to be worn. Menz and Morris (2005) recommend that footwear assessment should take place when assessing the needs of elderly patients as ill fitting footwear was a prevalent problem for this population. More recently Mickle et al, (2009) reported that the available shoes for the elderly population was limited, increasing the risk of injury. This report also indicated that the manufacture of such shoes was inadequate for females as the shoes manufactured were a scaled down version of the lasts used for gentlemans shoes leading to compromised toe box and problems with fitting.

Footwear style and choice do play a role in injury rehabilitation and it is an essential therapy to be utilised when treating patients with common musculoskeletal complaints which have been associated with poor fitting footwear (Dilnot et al, 2003; Yu et al, 2002). Over the centuries increases in heel height and narrowing toe boxes have been blamed for causing musculoskeletal injury (Menz et al, 2005; Luo et al, 2009). Furthermore, previous research indicates that the incidences of forefoot neuroma's are higher in females who wear shoes with a narrow toe box (Menz et al, 2008; Balint et al, 2003). Lateral compression at the forefoot alters metatarsal function compressing the nerve causing an impingement injury. Altering footwear style in the early stages of the complaint relieves symptoms and prevents further development of scars (Llanos et al, 1999).

It is known that plantar heel pain can be aggravated when footwear changes the foot position and function. Hence symptoms do not resolve when inappropriate footwear is worn. Addressing heel height, either by reducing or increasing, will alter the mechanics of the Achilles tendon and plantar fascia which are well documented as being the primary tissues involved in patients who have plantar heel pain (Burnfield et al, 2004; Rajput and Abboud, 2004). 
Forefoot equinus, a foot shape that is commonly observed, presents when the forefoot lies in a plantarflexed position to the rearfoot. (Figure 3) When placed in flat shoes this actively dorsiflexes the midtarsal joints jamming and compacting the metatarsals against the cuneiforms. Over time continual stress and repetitive trauma damages the joint leading to the $1^{\text {st }}$ stages of osteoarthritis. Wearing a shoe that has a suitable gradient in the shank (to accommodate for the lowering of the forefoot) and appropriate heel height will reduce the dorsiflexing moment and relieve any compression on the midtarsal joint complex. A heel over the height of $5 \mathrm{~cm}$ is thought to compromise muscle action and lower back stability. Murley et al, (2009) reviewed the influence of footwear on muscle activity and found that changes in fatigue occurred in heeled shoes particularly in the gastrocnemius muscle and lower back muscles. Heeled

\section{Figure 3}

Figure 3 Forefoot Equinus foot shape, the forefoot lies lower than the rearfoot.

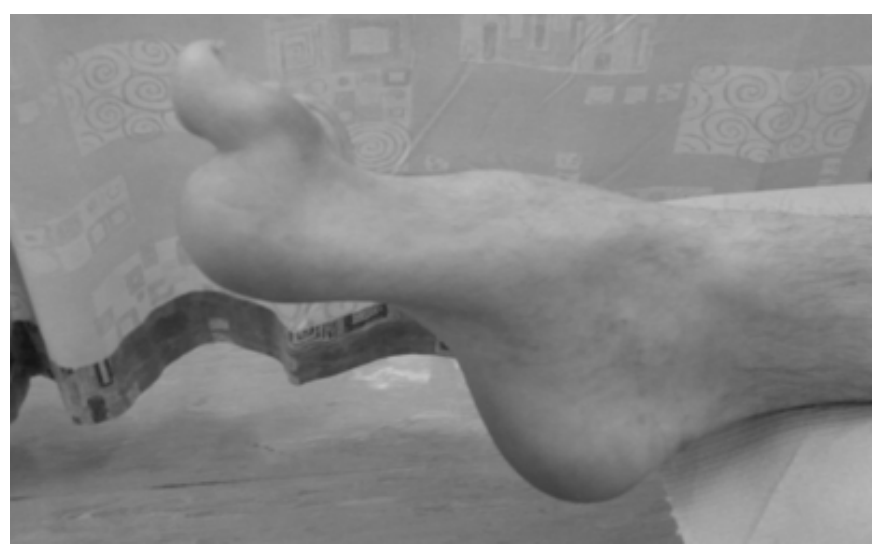

shoes are well documented as being detrimental on stability, muscle and tendon length, pelvic alignment and forefoot pressure (Hewitt et al, 2009; Speksnijde et al 2005; Lee et al, 2001).

\section{DISCUSSION}

The choice of footwear can be debilitating if an incorrect style and type is worn. Changing this to suitable shoes limits the progression of common foot deformities and reduces discomfort from superficial irritation. Furthermore, choosing to wear an appropriate pair of shoes initially could help in the prevention of general foot health complaints. Therapeutic footwear will continue to develop to aid and rehabilitate those who have significant systemic disease that inhibits sensation, balance, gait permutation and are unable to wear mass produced shoes.

The less complex problems that the general population suffer from are currently not as simple to manage with footwear. The prospect of being able to purchase footwear from a high street retailer specific to your needs, either preventative or therapeutic, should be a feasible option for people over the next decade.

\section{References}

r-0. Bálint,G. Korda,J. Hangody,L. Bálint, P. Foot and ankle disorders. Best Practice \& Research Clinical Rheumatology, 2003; 17(1):87-111

r-1. Benvenuti F. et. al, Foot pain and disability in older persons. J. Am Geriatr Soc ,1995; 43(5):479-484

r-2. Burnfield, JM. Few,CD. Mohamed, OS. Perry, J. The influence of walking speed and footwear on plantar pressures in older adults. Clinical Biomechanics, 2004; 19(1) :78-84

r-3. Bus,SA. Valk, GD. van Deursen, RW. Specific guidelines on footwear and offloading. Diabetes/Metabolism Research and Reviews, 2008(a); 24 (S1):S192 - S193 r-4. Bus,SA. Valk, GD. van Deursen, RW. Armstrong, DG. Caravaggi, C.Hlavacek, P. Bakker, K. Cavanagh, PR. The effectiveness of footwear and offloading interventions to prevent and heal foot ulcers and reduce plantar pressure in diabetes: a systematic review. Diabetes/Metabolism Research and Reviews, 2008(b); 24 (S1) :S162 - S180 r-5. Bus SA, van Deursen RW, Kanade RV, Wissink M, Manning EA, van Baal JG, Harding KG. Plantar pressure relief in the diabetic foot using forefoot offloading shoes. Gait Posture, 2009; 29(4):618-22

r-6. Decker, L. Houser, JJ. Noble, JM. Karst, GM. Stergiou, M. The effects of shoe traction and obstacle height on lower extremity coordination dynamics during walking. Applied Ergonomics, 2009;40 (5) 895-903

r-7. Dilnot, MC. Michaud, TC. Plantar plate rupture.

Australasian Journal of Podiatric Medicine,2003;37 (2): 4346.

r-8. Fuller,E. Schroeder,S. and Edwards,J . Reduction of Peak Pressure on the Forefoot with a Rigid Rocker-Bottom Postoperative Shoe. J Am Podiatr Med Assoc, 2001; 91: 501-507

r-9. Hennessy,K. Burns,J. Penkala, S. Reducing plantar pressure in rheumatoid arthritis: A comparison of running versus off-the-shelf orthopaedic footwear. Clinical Biomechanics, 2007; 22 (8):917-923

r-10. Hewitt, L. Wilgress, S. Jennings, E. Chockalingam, N. Pandyan, A. The effect of heel height on static and dynamic balance. Footwear Science 2009; 1 (S1)39-41

r-11. Hutchins, S. Bowker,P.Geary, N. Richards,J. The biomechanics and clinical efficacy of footwear adapted with rocker profiles-Evidence in the literature.The Foot, 2009;19(3):165-170

r-12. Janisse,DJ and Janisse E, Shoe Modification and the Use of Orthoses in the Treatment of Foot and Ankle Pathology. J Am Acad Orthop Surg, 2008; 16 (3):152-158 r-13. Lee,CM. Jeong,EH. Freivalds, E. Biomechanical effects of wearing high-heeled shoes International Journal of Industrial Ergonomics, 2001; 28 (6) :321-326

r-14. Long,JT. Klein,JP, Sirota,NM. Wertsch,JJ. Janisse,DJ. and Harris, GF .Biomechanics of the double rocker sole shoe: gait kinematics and kinetics, J Biomech 2007; 40(13):2882-2890

r-15. Luo, G. Stergiou, P. Worobets, J. Nigg, B.

Stefanyshyn, D. Improved footwear comfort reduces oxygen consumption during running. Footwear Science, 2009; 1, (1):25-29

r-16. Menant, J. Steele,J. Menz,H. Munro,B. Lord,SL. 
Effects of walking surfaces and footwear on temporo-spatial gait parameters in young and older people

Gait \& Posture, 2008;29 ( 3) 392-397

r-17. Menz, HB. Morris, ME. Footwear Characteristics and

Foot Problems in Older People. Gerontology, 2005;

51:346-351.

r-18. Mickle, KJ. Munro, BJ. Lord, SL. Menz, HB. Steele, JR. Can Grandma wear Grandpa's shoes? Footwear science, 2009; 1 (S1);5-6

r-19. Murley, G. Landorf, K. Menz, H. Bird,A. Effect of foot posture, foot orthoses and footwear on lower limb muscle activity during walking and running: A systematic review Gait \& Posture, 2009; 29 (2):172-187

r-20. Myers,KA. Long,JT. Klein,JP Wertsch,JJ. Janisse,DJ. and Harris, GF. Biomechanical implications of the negative heel rocker sole shoe: gait kinematics and kinetics, Gait

Posture, 2006; 24(3):323-330

r-21. Nigg, B. Hintzen, S and Ferber, R. Effect of an

unstable shoe construction on lower extremity gait

characteristics. Clin Biomech, 2006; 21(1):82-88

r-22. Nolan,J. Nolan,M. Booth,A. Developing the nurse's role in patient education: rehabilitation as a case example. International Journal of Nursing Studies, 2001;

38(2):163-173

r-23. Nurse,MA. Hulliger,M. Wakeling,JM. Nigg,BM.

Stefanyshyn, DJ.Changing the texture of footwear can alter gait patterns. Journal of Electromyography and Kinesiology, 2005;15(5):496-506

r-24. Piaggesi A, Viacava P, Rizzo L, Naccarato G, Baccetti F, Romanelli M, Zampa V, Del Prato S. Semiquantitative analysis of the histopathological features of the neuropathic foot ulcer: effects of pressure relief. Diabetes Care. 2003; 26(11):3123-8

r-25. Rajput, B. Abboud, RJ. Common ignorance, major problem: the role of footwear in plantar fasciitis. The Foot, 2004; 14(4):214-218

r-26. Romkes,J. Rudmann, C. and Brunner, R. Changes in gait and EMG when walking with the Masai Barefoot Technique, Clin Biomech, 2006; 21(1):75-81

r-27. Speksnijder,CM. Rieny JH. vd Munckhof, Sjors AF. Moonen,CM. Geert HI. Walenkamp,M. The higher the heel the higher the forefoot-pressure in ten healthy women. The Foot, 2005; 15(1):17-21

r-28. Tyrell W, Carter G, Therapeutic Footwear, A Comprehensive Guide. Churchill Livingstone 2009; ISBN : 978-0-443-06883-6 r-29. Willems,T.De Clercq,D.Delbaere,K.

Vanderstraeten,G.De Cock,A.Witvrouw,E. A prospective study of gait related risk factors for exercise-related lower leg pain. Gait \& Posture, 2006; 23 (1) :91-98 r-30. Williams, AE. and Nester,CJ. Patient perceptions of stock footwear design features, Prosthetics and Orthotics International, 2006;30:61-71 r-31. Wu,W. Rosenbaum, D. and Su,F. The effects of rocker sole and SCAH heel on Kinematics of Gait, Med Eng Phys 2004;26: 639-646

r-32. Yu GV, Judge MS, Hudson JR, Seidelmann FE.

Predislocation syndrome. Progressive subluxation/dislocation of the lesser metatarsophalangeal joint. Journal of the American PodiatricMedical Association, 2002. 92;(4):182-199 


\section{Author Information}

Helen Branthwaite

Faculty of Health, Staffordshire University

\section{Nachiappan Chockalingam}

Faculty of Health, Staffordshire University 